\section{Optical microangiography provides depth-resolved images of directional ocular blood perfusion in posterior eye segment}

\author{
Ruikang K. Wang, ${ }^{\mathrm{a}, *}$ Lin An, ${ }^{\mathrm{a}}$ Spencer Saunders, ${ }^{\mathrm{a}}$ and \\ David J. Wilson ${ }^{\mathbf{b}}$ \\ ${ }^{\mathrm{a}}$ Oregon Health \& Science University, Department of \\ Biomedical Engineering, 3303 South West Bond Avenue, \\ Portland, Oregon 97239 \\ ${ }^{\mathrm{b}}$ Oregon Health \& Science University, School of Medicine, \\ Casey Eye Institute, 3303 South West Bond Avenue, \\ Portland, Oregon 97239
}

\begin{abstract}
In this paper, we demonstrate that the optical microangiography (OAMG) is capable of depth-resolved imaging of directional blood perfusion within both retinal and choroid in the posterior segment of human eye. The study uses an OMAG system operating at $840 \mathrm{~nm}$ with an imaging speed at 27,000 A-scans per second. Sequentially registered multiple OMAG projection maps of small areas $(\sim 1 \mathrm{~mm} \times 1 \mathrm{~mm})$ are combined to provide directional blood flow images for a larger field of view. It takes $\sim 3.7 \mathrm{sec}$ to image a small area $\left(1 \times 1 \mathrm{~mm}^{2}\right)$, and $\sim 2.5 \mathrm{~min}$ for a larger field $\left(3 \times 3 \mathrm{~mm}^{2}\right)$. Finally, we show superior performance of OMAG in providing functional images of capillary level microcirculation at different depths of retina and choroid that correlate well with the standard retinal pathology. @ 2010 Society of Photo-Optical Instrumentation Engineers. [DOI: 10.1117/1.3353958]
\end{abstract}

Keywords: optical microangiography; optical coherence tomography; ophthalmology; retinal and choroidal blood perfusion.

Paper 09565LR received Dec. 19, 2009; revised manuscript received Feb. 4, 2010; accepted for publication Feb. 9, 2010; published online Mar. 17, 2010.

There is a growing body of evidence suggesting that vascular pathology plays an important role in the etiology and progression of a number of ophthalmic diseases, e.g., glaucoma, diabetic retinopathy and age-related macular degeneration (AMD). In glaucoma, the increased intraocular pressure may change the ocular blood flow, which could lead to the tissue ischemia and finally the cell death. ${ }^{1}$ For the diabetic retinopathy, the clinical evidence has shown reduced ocular capillary density gives rise to the enlargement of foveal avascular zone and the perifoveal inter-capillary region. ${ }^{2}$ The choroidal neovascularization in AMD may be the direct result of decreasing in choroidal blood flow. ${ }^{3}$ In these pathological conditions, a better visualization of the depth-resolved ocular vasculature characteristics, together with morphological features may provide important diagnostic information.

Optical microangiography (OMAG) is a recently developed novel imaging technique that produces $3 \mathrm{D}$ images of

*Address all correspondence to: Ruikang K. Wang. Tel: 503 4189317; Fax: 503 4189311; E-mail: wangr@ohsu.edu. dynamic blood perfusion within micro-circulatory tissue beds at an imaging depth up to $2.0 \mathrm{~mm}^{4-7}$ OMAG produces imaging contrasts via endogenous light scattering from moving particles (e.g., flowing blood cells within open vessels), thus, no exogenous contrast agents are necessary. OMAG has its origin in full range complex Fourier domain optical coherence tomography (OCT), ${ }^{8,9}$ but it innovatively analyzes the spatial frequencies of the spatial and time-varying spectral interferograms. $^{4-7}$ In essence, OMAG mathematically maps the backscattered optical signals from the moving particles into one image - that is the blood flow image - while it simultaneously maps the backscattered optical signals from the static particles into a second image, which is the microstructural image. These distinct features of OMAG provide us a unique ability to perform comprehensive measurements of the morphological and functional parameters of blood perfusion within a scanned tissue volume. OMAG has been successfully demonstrated for imaging cerebral blood flow in mice and rat $^{4,5}$ and, recently, the ocular blood flow. ${ }^{6}$ However, the previous versions of OMAG only provide the reflectivity measurements of the flowing blood in the patent vessels, particularly for retina. In this paper, we report the use of OMAG imaging technology to obtain high resolution and depth resolved images of directional ocular blood flows.

The OMAG system setup used to achieve directional ocular blood flow imaging is similar to that used in our previous work. ${ }^{7}$ Here we briefly describe its main parameters. The system used a superluminescent diode as the light source, with a central wavelength of $842 \mathrm{~nm}$ and bandwidth of $46 \mathrm{~nm}$ that provides a $\sim 8 \mu \mathrm{m}$ axial resolution in the air. For maintaining high resolution imaging of blood vessels, the area of each OCT scan was set relatively small. In the lateral direction (B scan), we captured $1000 \mathrm{~A}$-scans, covering a size of $\sim 1 \mathrm{~mm}$ on retina, and in the $\mathrm{C}$-scan (elevational direction), we captured $100 \mathrm{~B}$-scans over a size of also $\sim 1 \mathrm{~mm}$. The imaging rate was $27 \mathrm{kHz}$ for A scan, and 27 frames per second (fps) for B scan, which took about $3.7 \mathrm{sec}$ to complete a C scan, representing a physical volume of $\sim 1 \times 1 \times 2(x-y-z) \mathrm{mm}^{3}$. To achieve a larger field of view of fundus vasculatures, we sequentially captured $9 \mathrm{C}$-scans at different locations on the posterior segment of the eye. Between every two C-scans, we introduced $\sim 0.1 \mathrm{~mm}$ overlap area to make sure no blank region occurred when reconstructing the wide field images. In between $\mathrm{C}$-scans, the volunteer was instructed to close his eye for $15 \mathrm{~s}$ for a rest and prepare for the next scan. Hence, it took about 2.5 minutes to acquire one blood perfusion map, representing a scan volume of $\sim 3 \times 3 \times 2(x-y-z) \mathrm{mm}^{3}$.

When configured in morphological imaging mode, OMAG imaged the intra-retinal and choroidal microstructures, with image quality similar to that of state of the art OCT systems. Figure 1 gives one such example that was obtained from the macular region of a healthy volunteer. Note that in this experiment, the B scan was set to cover $\sim 7 \mathrm{~mm}$ on the retina. It is clear that OMAG is capable of visualizing the architectural morphology of the intraretinal and choroidal layers that correlate well with the intraretinal anatomy. For blood flow imaging, OMAG treats the microstructures as if they were noises to the system. This is achieved by the modified Hilbert transformation on the captured spatial-varying spectral interfero-

1083-3668/2010/15(2)/020502/3/\$25.00 @ 2010 SPIE 


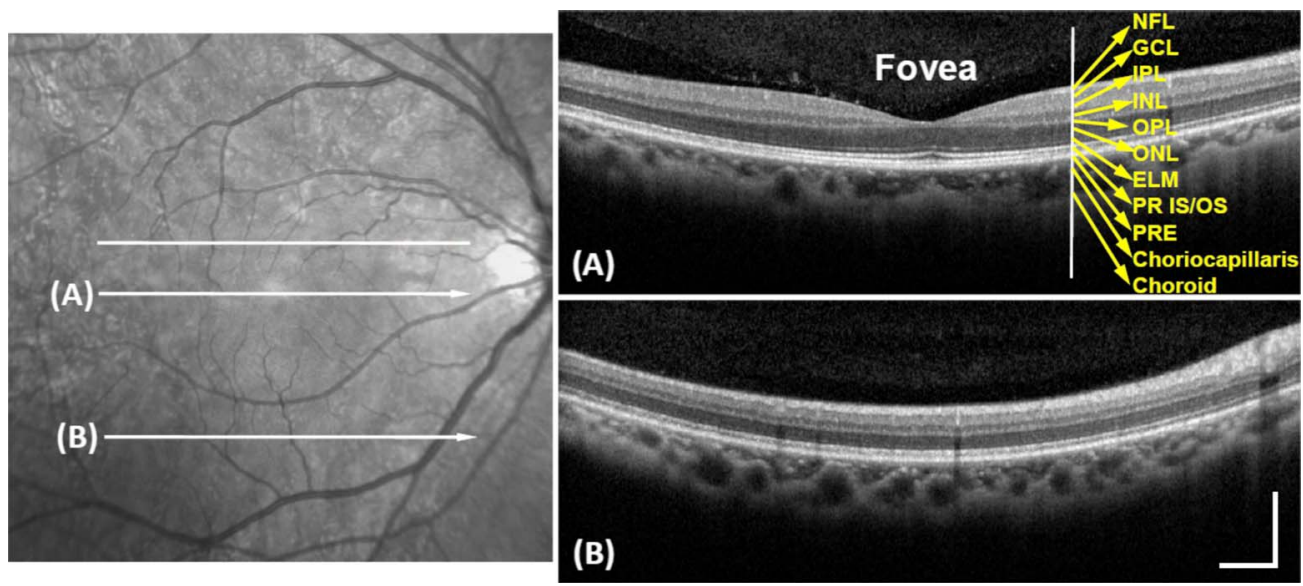

Fig. 1 When configured in structural imaging mode, OMAG imaged detailed morphological features within retina and choroid. Shown in the left is the standard fundus camera image in which the lines marked (a) and (b) are the locations for OMAG scan to give the intra-retinal and choroidal architectures shown in the right. NFL: Nerve fiber layer, GCL: ganglion cell layer, IPL: inner plexiform layer, INL: inner nuclear layer, OPL: outer plexiform layer, ONL: outer nuclear layer, ELM: external limiting membrane, PR IS/OS: photoreceptor inner and outer segments, RPE: retinal pigment epithelium. Bar $=500 \mu \mathrm{m}$.

grams to separate the blood flow signals from the static tissue signals. The detailed description of this method has been reported previously. ${ }^{4,5}$ Here we introduce the directional imaging using OMAG. The B-scan interference signal captured by the OCT system can be written as:

$$
\begin{aligned}
I(t, k)= & 2 S(k) E_{R}\left[\int_{-\infty}^{\infty} a(z, t) \cos (2 k n(t) z) d z\right. \\
& \left.+a\left(z_{1}, t\right) \cos \left[2 k n(t)\left(z_{1}-v t\right)\right]\right],
\end{aligned}
$$

where $k$ is the wavenumber; $t$ is the timing when a A-line was captured. $E_{R}$ is the light reflected from the reference mirror; $S(k)$ is the spectral density of the light source used; $n$ is the refractive index of tissue; $z$ is the depth coordinate; $a(z, t)$ is the amplitude of the back scattered light; $v$ is the velocity of moving blood cells in a blood vessel, which is located at depth $z_{1}$. Because the light backscattered from the sample is quite weak compared to the light reflected from the reference mirror, here we do not consider the self cross-correlation between the light backscattered from different positions within the sample. We also do not consider the DC signals because they do not contribute to useful OMAG signals. The first term in Eq. (1) represents the spatial frequency components from the static tissue elements, which can be used to provide the static structural information of the sample, such as that shown in Fig. 1. The second term is the Doppler beating signal, determined by the moving blood cells. If we first apply high pass filtering with an appropriate selection of cut off frequency to exclude the structural information, and then apply the Hilbert transformation along B-scan direction, the result will be:

$$
\begin{aligned}
I(k, t)_{\mathrm{DFlow}}= & 2 S(k) E_{R} a\left(z_{1}, t\right)\left[\operatorname { c o s } \left[2 k n(t)\left(z_{1}-v t\right)\right.\right. \\
& -j \sin \left[2 k n(t)\left(z_{1}-v t\right)\right]
\end{aligned}
$$

when $v>0$ (i.e., the blood cells moving towards the incident probe beam direction). Whereas when $v<0$ (i.e., the blood cells moving away from the incident probe beam direction), Eq. (2) becomes:

$$
\begin{aligned}
I(k, t)_{\mathrm{DFlow}}= & 2 S(k) E_{R} a\left(z_{1}, t\right)\left[\operatorname { c o s } \left[2 k n(t)\left(z_{1}-v t\right)\right.\right. \\
& +j \sin \left[2 k n(t)\left(z_{1}-v t\right)\right] .
\end{aligned}
$$

Mathematically, Eq. (3) is clearly the complex conjugate of Eq. (2). Therefore, according to Ref. 4, the directional blood flow is discriminated. The optical signals that represent the blood cells moving towards the probe beam will be situated at the positive output plane of OMAG, while those from the blood cells moving away from the probe beam direction will be located at the negative plane.

In the case of larger field of view imaging (multi-C scans), a serious problem caused by the involuntary movement of the human head and eye is the displacement between adjacent B-scans. We used the cross correlation between adjacent OMAG flow images to estimate both the axial and the lateral displacements between adjacent B-scans. Another main problem for in vivo visualizing human ocular perfusion is the bulk motion artifact within each B scan, also introduced by the human eye and head movements. To eliminate this bulk motion artifact, we adopted the phase compensation method described in Ref. 7. The histogram based phase estimation method $^{6}$ was employed to better estimate phases used in the phase compensation method. These motion compensation methods worked well to remove/minimize the subject movement artifacts presented in the OMAG flow images.

Because of its high sensitivity to the flow, OMAG delivers superior performance for imaging the blood vessel networks both in retina and choroid. Especially after compensation for the motion artifacts, even the capillary networks can be effectively imaged. The depth-resolved imaging results for directional blood perfusion within an area of $\sim 3 \times 3 \mathrm{~mm}^{2}$ centered around fovea in the macular region is shown in Fig. 2, where the scanning area for flow imaging is box-marked in the top microstructural image. Because of the depth-resolved nature, we are able to separate the blood flows at different land-mark depths. To do this, we first used the segmentation 


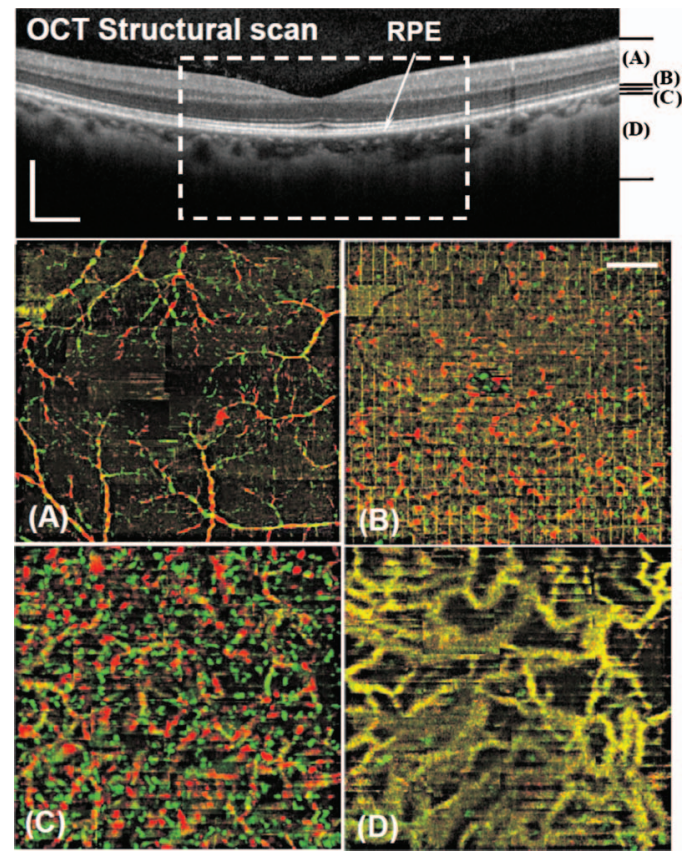

Fig. 2 Shown in the top is one representative OMAG/OCT structure image where the projection maps of the blood flows within the marked depth-regions generated by OMAG are given in the bottom. The red color represents the positive flow (towards the probe beam) and the green the negative flow. Depth resolved directional projection maps are for (a) retina; (b) within a layer from 0 to $30 \mu \mathrm{m}$ below RPE; (c) within a layer from 30 to $100 \mu \mathrm{m}$ below RPE; and (d) within a layer from 100 to $500 \mu \mathrm{m}$ below RPE. Bar $=500 \mu \mathrm{m}$.

algorithm described in Ref. 6 to identify the RPE layer. We then treat the OMAG flow signals above the RPE layer as from the retinal vessels, while those below the RPE layer from the choroidal vessels. According to the well-known reference 10, the choroidal vessels in the macular area are not specialized like those in the retina. The arteries pierce the sclera around the optic nerve and fan out to form three vascular layers in the choroid: outer arteries and veins, medial arterioles and venules, and inner capillary bed (near RPE layer). The directional blood flows within retina is shown in Fig. 2(a). The blood flows within a layer from 0 to $30 \mu \mathrm{m}$ below RPE is given in Fig. 2(b), which closely represents the inner capillary beds where the flow is slow, while the medial arterioles and venules are mostly confined within a layer from 30 to $100 \mu \mathrm{m}$ below RPE (Fig. 2(c)). Fast blood flows within the arteries and venues in deeper choroidal region is given in Fig. 2(d), where because the flow is too fast that exceeds OMAG directional discrimination capability, the color appears yellow in OMAG directional flow image. These obser- vations are correlated well with the standard retinal pathology. ${ }^{10}$ Note that the vertical artifacts, particularly in Fig. 2(b) were caused by the fixed electronic noise in the system, which is currently under development to minimize.

In summary, we used the OMAG technology to achieve imaging of ocular perfusion both in retina and choroid by stitching 9 small areas of $\mathrm{C}$-scans. The datasets provided high resolution blood vessel maps down to the capillary level resolution, together with important information about the directions of blood flow. We have shown the projection images of directional blood perfusion at different land-mark depths, and the superior imaging results promise the future clinical applications for OMAG.

This study was supported in part by research grants from the National Institutes of Health (R01 HL093140-01, R01 EB009682-01, and R01 DC010201-01), and an unlimited grant from Research to Prevent Blindness. Mr. Saunders would like to acknowledge the generous support of the Pete and Rosalie Johnson Internship Program in the School of Chemical, Biological, and Environmental Engineering at Oregon State University. OptoVue has licensed technology from OHSU of which Dr. Wang is an inventor. The technology is used in this research, which however did not receive support from OptoVue.

\section{References}

1. J. Flammer, S. Orgul, V. P. Costa, N. Orzalesi, G. K. Krieglstein, L. M. Serra, J.-P. Renard, and E. Stefansson, "The impact of ocular blood flow in glaucoma," Prog. Retin Eye Res. 21, 359-393 (2002).

2. V. Patel, S. Rassam, R. Newsom, J. Wiek, and E. Kohner, "Retinal blood flow in diabetic retinopathy," Br. Med. J. 305, 678-683 (1992).

3. E. Friedman, "A hemodynamic model of the pathogenesis of agerelated macular degeneration," Am. J. Ophthalmol. 124, 677-682 (1997).

4. R. K. Wang, S. Jacques, Z. Ma, S. Hurst, S. Hanson, A. Gruber "Three dimensional optical angiography," Opt. Express 15, 40834097 (2007).

5. R. K. Wang, and S. Hurst "Mapping of cerebrovascular blood perfusion in mice with skin and cranium intact by optical microangiography at $1300 \mathrm{~nm}$ wavelength," Opt. Express 15, 11402-11412 (2007).

6. L. An, and R. K. Wang, "In vivo volumetric imaging of vascular perfusion within human retina and choroids with optical microangiography," Opt. Express 16, 11438-11452 (2008).

7. R. K. Wang and L. An, "Doppler optical micro-angiography for volumetric imaging of vascular perfusion in vivo," Opt. Express 17(11), 8926-8940 (2009).

8. R. K. Wang, "In vivo full range complex Fourier domain optical coherence tomography," Appl. Phys. Lett. 90, 054103 (2007).

9. R. K. Wang, "Fourier domain optical coherence tomography achieves full range complex imaging in vivo by introducing a carrier frequency during scanning," Phys. Med. Biol. 52, 5897-5907 (2007).

10. H. R. Zhang, "Scanning electron-microscopic study of corrosion casts on retinal and choroidal angioarchitecture in man and animals," Prog. Retin Eye Res. 13, 243-270 (1994). 